\title{
A METHOD FOR REAL-TIME RENDERING OF WATER DROPLETS TAKING INTO ACCOUNT INTERACTIVE DEPTH OF FIELD EFFECTS
}

\author{
Tomoya Sato, Yoshinori Dobashi, Tsuyoshi Yamamoto \\ Graduate School of Engineering, Hokkaido University \\ \{tom, doba, yamamoto\}@nis-ei.eng.hokudai.ac.jp
}

\begin{abstract}
Many researchers have been studying computer graphics simulations of natural phenomena. One important area in these studies is the animation of water droplets, whose applications include drive simulators. Because of the complexity of shape and motion, the animation of water droplets requires long calculation time. This paper proposes a method for real-time animations of water droplets running down on a glass plate, using graphics hardware. The proposed method takes into account depth of field effects, and makes it possible to change the focal point interactively depending on a point in the center of the scene being observed.
\end{abstract}

Keywords: Animation, Water Droplets, Real-Time Rendering, Graphics Hardware, depth of field Effects

\section{Introduction}

Recently, many computer graphics researchers have studied simulations of various natural phenomena such as clouds, water, terrain, and flame. Their research results have been used in entertainment applications such as computer games. Among those simulations, the animation of water droplets is indispensable for drive simulators, for rendering scenes viewed through a windowpane on a rainy day, and for computer games. For displaying the animation of water droplets, several methods have been presented [2][3][4][5]. These methods can generate realistic animation. However, because the motion of water droplets is complex and ray tracing is used for rendering, there is a problem of long calculation time. In making an animation, it is important to create an image as fast as possible, because we must generate a number of images.

The original version of this chapter was revised: The copyright line was incorrect. This has been corrected. The Erratum to this chapter is available at DOI: 10.1007/978-0-387-35660-0_65 
In recent years, however, increased processing speed of graphics hardware have enabled traditionally time-consuming process to be performed much faster. This paper proposes a hardware-accelerated method for real-time animation of water droplets. Additionally, we introduce depth of field effects. These effects mimic that whereby an eye focused on an object sees the objects placed out of the field of focus as blurred. Our method makes it possible to change the focal point interactively depending on the point in the center of scene being observed.

\section{Previous Work}

A method of animating the flow of small amounts of shallow water like water droplets was developed by Kass et al. [1]. This method enables the efficient display of animated rain falling on a concave surface. However, it is difficult to apply their method to the animation of water droplets, which is our purpose, because of the absence of interfacial dynamics.

A method of animating the flow of water droplets was developed by Kaneda et al. [2][3][4][5]. In it, the interfacial dynamics are taken into account [2]. That is, when water droplets run down on a surface, some amount of water remains behind because of the wetting phenomena. To simulate the motions of water droplets, a discrete surface model is developed. That is, a glass plate was divided into a mesh. Furthermore, improved methods were presented [3][4][5]. In these papers, the method was extended to a curved surface, taking into account influence of wind, windshield wiper and depth of field effects. Moreover, images were created faster by interpolating pre-computed images of water droplets. Though these methods enable us to generate a realistic animation, the computational cost is still great because the rendering method is based on ray tracing. They generate one image in about half a minute. Therefore, they cannot be applied to a real-time animation.

\section{Proposed Method}

Our target is a drive simulation that can represent driving on a rainy day. This paper proposes a method of animating water droplets running down on a windshield of a car. As shown in Fig. 1, we assume the viewpoint is inside the car. The movements of water droplets are computed by using the method described in [2]. This paper focuses on a hardware-accelerated method for rendering water droplets and depth of field effects. First, the basic idea of the proposed method is described. Then, details of the method are explained. 


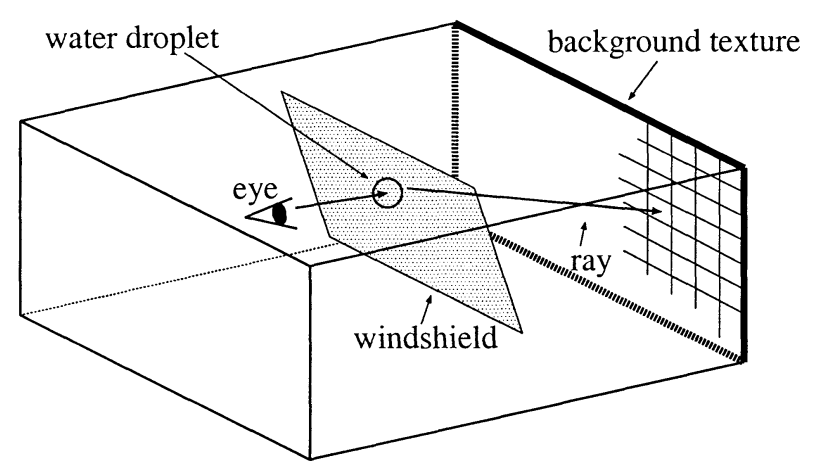

Figure 1. Model of our method.

\subsection{Basic Idea}

Water droplets are assumed to be hemispherical (Fig. 2). The hemisphere is approximated by polygons. Then water droplets are rendered by graphics hardware. The color of the water droplet is computed by mapping a background texture as shown in Fig. 2. This makes it possible to utilize graphics hardware. However, the rendering time is increased when the number of droplets is large. In general, the size of the water droplet is very small. So, the appearances of water droplets are almost the same for all droplets that are close to each other. The proposed method makes use of these properties to create images faster. First, the windshield is divided into a mesh (Fig. 3). Next, textures of water droplets are created by rendering the water droplets placed at the lattice points of the mesh. We call these textures "water droplet textures". Then the water droplet at an arbitrary position is rendered by drawing a quadrilateral onto which the water droplet textures are mapped. By using this method, one quadrilateral is sufficient for rendering one water droplet. This reduces the rendering time. After rendering the background and water droplets, the final image is generated by providing the depth of field effects.

\subsection{Background Texture}

Intersection points between a viewing ray and objects must be computed for the precise rendering of scenes including transparent objects such as water droplets and the windshield. However, this process is computationally very expensive. We make use of the idea of the environmental refraction mapping [6]. First, the objects viewed through the windshield are rendered without the water droplets. We ignore the 


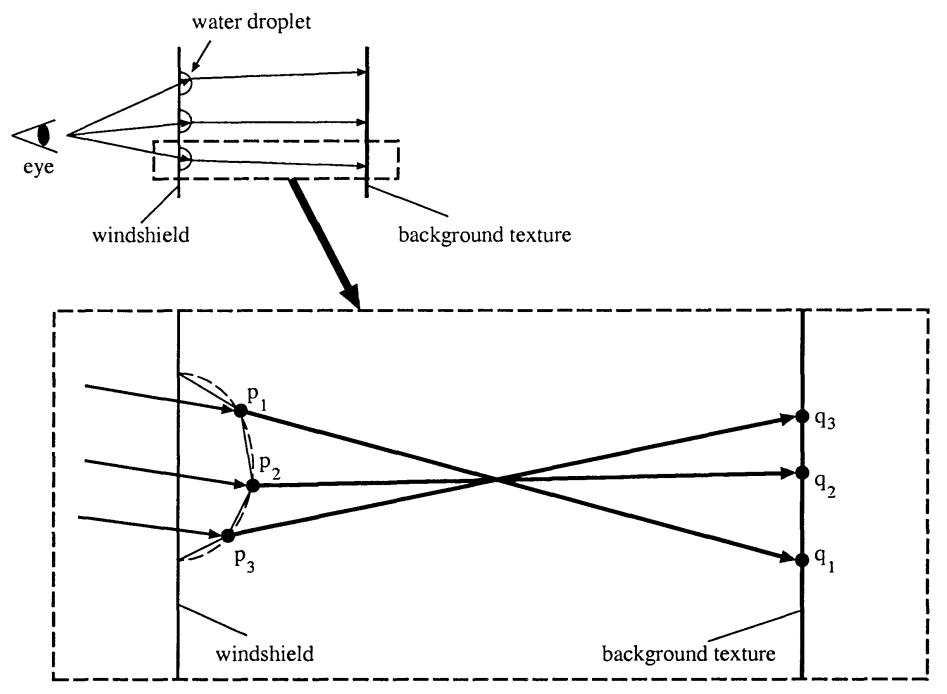

Figure 2. Calculation of water droplet color.

refraction of light due to the windshield since it is very thin. The resulting image is stored as the background texture. Then water droplets are rendered by mapping the background texture onto the hemispheres representing the water droplets (Fig. 1). In this paper, we use photo images as the background texture. In this case, we can omit the process for rendering the objects viewed through the windshield without the water droplets.

\subsection{Water Droplet Texture}

The water droplet textures are created and stored for each lattice point (Fig. 3). For creating the water droplet textures, the water droplets are represented by the polygonized hemispheres (Fig. 2). The textures are created by the following procedures. First, the reference point of the viewer is set to the lattice point. The viewing angle is set so that it is tightly fits the bounding volume of the water droplet. The reflection of light from the surface of the water droplet can be ignored since its contribution to the pixel intensity is very small. Then the water droplet is rendered by drawing the polygonized hemisphere. The background texture described in the previous section is mapped onto the hemisphere. The mapping process is as follows. As shown in Fig. 2, let us assume a viewing ray passing through a vertex $p_{i}$ of the polygonized hemisphere. The ray intersects the background at point $q_{i}$ after the refraction due to the water droplet. The background texture is 
mapped onto the water droplet so that the intersection $q_{i}$ corresponds to the vertex $p_{i}$. After rendering the water droplet, the resulting image is stored as the water droplet texture. These processes are repeated for all lattice points.

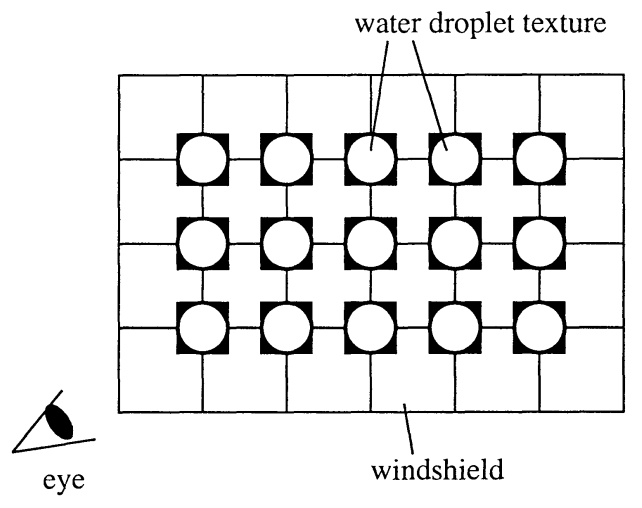

Figure 3. Texture Generation.

\subsection{Rendering Water Droplets}

The water droplets are displayed by using the water droplet textures described in section 3.3. For each water droplet, four lattice points, $i, j, k, l$, that surround the water droplet are determined. Then a quadrilateral is placed at the position of the water droplet. The quadrilateral is called a billboard. The size of the billboard is the same as the diameter of the water droplet. Four water droplet textures that correspond to each of lattices, $i, j, k, l$, are mapped onto the billboard by using a multi-texture function supported by hardware.

\subsection{Depth of Field Effects}

The final image is generated by providing the depth of field effects. In the real world, when the eye is focused on an object, other objects out of the field of focus are blurred. Taking into account the depth of field effects, we can create more realistic images. Mulder et al. proposed a perception-based method for the depth of field effects [7]. Heidrich et al. proposed an image-based method that simulates real lens systems for interactive computer graphics [8]. Their methods can generate the effects efficiently by using graphics hardware. These methods, however, are not fast enough for real-time rendering. Therefore, we decided on a method using simple convolution filters [9]. 
First, after rendering the background image $\left(\mathrm{I}_{0}\right)$, depth values of all pixels are read from the frame buffer. Then, the image $\mathrm{I}_{0}$ is blurred by using the two following filters:

$$
f_{1}=\left[\begin{array}{lll}
1 & 2 & 1 \\
2 & f & 2 \\
1 & 2 & 1
\end{array}\right](f \geq 4), \quad f_{2}=\left[\begin{array}{lll}
1 & 1 & 1 \\
1 & f & 1 \\
1 & 1 & 1
\end{array}\right](f \geq 1)
$$

This results in two images, one blurred by $f_{1}$ and the other by $f_{2}$. The blurred effects by $f_{2}$ are stronger than those by $f_{1}$. Let us denote the image blurred by $f_{1}$ as $I_{1}$ and that blurred by $f_{2}$ as $I_{2}$. Then, the difference $d(x, y)$ between a depth value at the view reference point and at each pixel is calculated. Blurred background image $\mathrm{I}_{b}$ is created by using the following equation:

$$
\mathrm{I}_{b}(x, y)= \begin{cases}\mathrm{I}_{0}(x, y) & \text { if } d(x, y) \leq \varepsilon_{0} \\ \mathrm{I}_{1}(x, y) & \text { if } \varepsilon_{0}<d(x, y) \leq \varepsilon_{1} \\ \mathrm{I}_{2}(x, y) & \text { if } \varepsilon_{1}<d(x, y)\end{cases}
$$

where $\varepsilon_{0}$ and $\varepsilon_{1}$ are thresholds specified by the user $\left(\varepsilon_{0}<\varepsilon_{1}\right)$. This means that there are no blurring effects around the view reference point and that blurring effects of two phases appear as $d(x, y)$ becomes large. In other words, we can approximately render the depth of field effects.

Next, the image of the water droplets only is created $\left(\mathrm{I}_{w}\right)$. For each pixel of the image $\mathrm{I}_{w}$, an alpha value is stored together with the color of the water droplets. The alpha value is given by:

$$
\alpha(x, y)= \begin{cases}1 & \text { (if water droplets exist at pixel }(x, y)) \\ 0 & \text { (otherwise) }\end{cases}
$$

Using the alpha value and the filter $f_{2}$ described above, the blurred image $\mathrm{I}_{w}^{\prime}$ is created from $\mathrm{I}_{w}$. The water droplets are on the windshield and, in general, the view reference point is far from the windshield. So, we apply $f_{2}$ because blurred effects are stronger. $\mathrm{I}_{w}^{\prime}$ is created by using the following equation:

$$
\mathrm{I}_{w}^{\prime}(x, y)=\frac{\sum_{i=-1}^{1} \sum_{j=-1}^{1} \alpha(x+i, y+j) \mathrm{I}_{w}(x+i, y+j)}{\sum_{i=-1}^{1} \sum_{j=-1}^{1} f_{2}(i, j) \alpha(x+i, y+j)} .
$$

The alpha value prevents the pixel color from blending with the black pixels where no water droplets exist. Without alpha value, the pixel intensity is decreased. Furthermore, we apply $f_{2}$ for alpha values by using the following equation: 


$$
\alpha^{\prime}(x, y)=\frac{\sum_{i=-1}^{1} \sum_{j=-1}^{1} f_{2}(i, j) \alpha(x+i, y+j)}{\sum_{i=-1}^{1} \sum_{j=-1}^{1} f_{2}(i, j)} .
$$

$\alpha^{\prime}$ is used for blending the background image $\mathrm{I}_{b}$ and the water droplet image $\mathrm{I}_{w}^{\prime}$. The final image $\mathrm{I}_{f}$ is created by blending $\mathrm{I}_{b}$ and $\mathrm{I}_{w}^{\prime}$ using the following equation:

$$
\mathrm{I}_{f}(x, y)=\left(1-\alpha^{\prime}(x, y)\right) \mathrm{I}_{b}(x, y)+\alpha^{\prime}(x, y) \mathrm{I}_{w}^{\prime}(x, y) .
$$

Thus, although our method is not physically correct, we can render the depth of field effects efficiently just by blending the background and water droplet images after filtering them. This means the depth of field effects can be interactively generated according to the view reference point.

\section{Examples}

In this section, we demonstrate the usefulness of the proposed method by applying it to the drive simulation on a rainy day. For creating the water droplet textures in section 3.3, the windshield is divided into an $11 \times 11$ mesh. The parameters $f$ of two convolution filters described in section 3.5 are 1 for $f_{1}$ and 5 for $f_{2}$. There are approximately 1,000 water droplets in each frame. The image resolution is $320 \times 240$. The animation is created on a PC (Athlon $900 \mathrm{MHz}$ ) with a NVIDIA GeForce3.

The animation of water droplets is created under the conditions described above. Figs. 4 and 5 show the images from the animation with and without the depth of field effects, respectively. In Fig. 4, the focal point can be changed interactively depending on the view reference point. By taking into account the depth of field effects, more realistic images are created. The frame rate of the animation is about $14 \mathrm{fps}$ with the depth of field effects and about 55 fps without the effects. When we use movies for background texture, the frame rate of animation is about $12 \mathrm{fps}$. This is because the background texture is replaced at each frame. These results demonstrate that our method can create realistic animation almost in real-time.

\section{Conclusions}

This paper proposed a method for the real-time animation of water droplets running down a glass plate, using graphics hardware. Our method takes into account the depth of field effects. The focal points can be changed interactively depending on the view reference point. Because 
it realizes the real-time animation of water droplets, our method enables the animation of water droplets in applications that requires real-time processing, such as drive simulations, and computer games.

What remains to be discussed is, first, acceleration of the processes. This is because animation generally requires $30 \mathrm{fps}$. For more accurate results, we must develop a faster method that taking into account the actual lens system, since we used simple convolution filters for the depth of field effects described in section 3.5.

\section{References}

[1] M. Kass, G. Miller, "Rapid, Stable Fluid Dynamics for Computer Graphics", Proc. of SIGGRAPH'90, pp. 49-57(1990).

[2] K. Kaneda, T. Kagawa, H. Yamashita, "Animation of Water Droplets on a Glass Plate", Proc. of Computer Animation '93, pp. 177-189(1993).

[3] K. Kaneda, Y. Zuyama, H. Yamashita, T. Nishita, "Animation of Water Droplet Flow on Curved Surface", Proc. of Pacific Graphics'96, pp. 50-65(1996).

[4] K. Kaneda, S. Ikeda, H. Yamashita, "Animation of Water Droplets Moving Down a Surface", The Journal of Visualization and Computer Animation, Vol. 10, No.1, pp. 15-26(1999).

[5] R. Matsumoto, K. Kaneda, H. Yamashita, "A Fast Rendering Method for Water Droplets Using an Image Interpolation", Visual Computing Workshop 2001, 2001.

[6] N. Greene, "Environment Mapping and Other Applications of World Projections", IEEE Computer Graphics and Applications, pp. 21-29(1986).

[7] J. Mulder, R. V. Liere, "Fast Perception-Based Depth of Field Rendering", Proc. of ACM VRST2000, 2000.

[8] W. Heidrich, P. Slusallek, H. Seidel, "An Image-Based Model for Realistic Lens Systems in Interactive Computer Graphics", Proc. of Graphics Interface '97, pp. 68-75(1997).

[9] P. Rokita, "Application of image processing techniques in computer graphics algorithms", Computer Networks and ISDN System 29, pp. 1705-1714(1997).

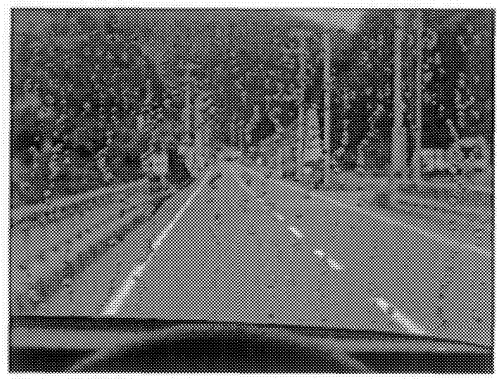

Figure 4. Example of an image with the depth of field effects.

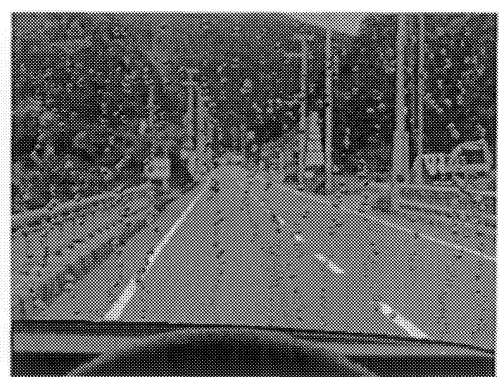

Figure 5. Example of an image without the depth of field effects. 